\title{
Mobile Radio Propagation along Mixed Paths in Forest Environment using Parabolic Equation
}

\author{
João F. de Souza, Fátima N. B. Magno, Zínia A. Valente, Jessé C. Costa, Gervásio P. S. \\ Cavalcante \\ Universidade Federal do Pará, furtado@ufpa.br, fnbm@ufpa.br, zinia@ufpa.br, \\ jesse@ufpa.br, gervasio@ufpa.br
}

\begin{abstract}
This paper calculates the propagation loss in mixed-path mobile communication using parabolic equation method. The implicit finite differences scheme of the Crank-Nicolson type is applied in order to get the solution of the parabolic equation. The propagation is considered in $15^{\circ}$ in the direction paraxial and the complex refractive index was considered what increases its precision. To validate the method, a radio propagation measurement campaign was carried out, in the county of Benfica, near the city of Belém, State of Pará. The frequency used was $900 \mathrm{MHz}$. The results were compared to the simulations made with the proposed method, which presented the best performance comparing to others works in literature. The loss predicted by this method had a mean error of $3.40 \mathrm{~dB}$ and standard deviation of $2.75 \mathrm{~dB}$ in relation to the measured data.
\end{abstract}

\section{Introduction}

For line-of-sight communication, cellular communication in particular current interest centers on radio-link performance, and how it is affected by wave attenuation, fading and co-channel interference. When vegetation, such as a forest, lies along the path of a radio performance is affected by strong multiscattering effects and imposes stringent constraints on system design. Therefore, accurate modeling of wave propagation behavior through foliage has attracted significant attentions in recent years for its applications in many civilian and military problems. The most important effect introduced by the forest is the excess signal attenuation (or path-loss) in addition to that due to free space propagation [1] - [3].

Several methods to model vegetation for propagation analysis have been presented in literature. Empirical and deterministic models are available, mainly aimed at characterizing the effects of vegetation and calculation of path loss. The empiric model are based in simple and direct formulas, very easy to apply, but on the other hand these models provide poor local-specific precision and they cannot predict parameters of the broad band of the communication channel. The deterministic models follow physical principles of propagation of electromagnetic waves - most popular they are ray tracing and the numerical solution of the equations of Maxwell.

One of the most reliable and widely methods is the Parabolic Equation (PE) Method, initially developed for the study of underwater acoustics problems and later on extended to tropospheric propagation. It has been proved that like troposphere parabolic equation in vegetation. The biggest advantage to using the PE method is that it gives a full-wave solution for the field in the presence of range-dependent environments. This method supplies an excellent combination of precision and efficiency for many problems of electromagnetic waves propagation [4].

The method presented in this paper is based on the parabolic equation (PE) using the finite difference scheme of Crank-Nicolson to calculate the path loss in a forest environment. A complex refractive index was considered in the studied environment and the propagation angle was considered in $15^{\circ}$ in the direction paraxial. In this formulation the backscattered field was neglected.

To validate the proposed method a measurement campaign was carried out in a mixed path in forest environment in Benfica, Belém - Pará. The frequency used was $900 \mathrm{MHz}$. The results of the simulation through the proposed method had been compared with the experimental data and models of BlomquistLadell's method, Multiray' s model and Okumura-Hata' s model.

This work is organized as it follows: the problem formulation, the parabolic equation (PE) method and the finite difference scheme of Crank-Nicolson, is described in section 2; the path loss models are shown in the section 3; the section 4 shows the results and the section 5 , the conclusion. 


\section{Problem Formulation}

Assuming propagation to be predominantly along the axis and the paraxial cone with a narrow-angle, $\theta$ as shown in Figure 1, the 2-D Parabolic Equation can be derived from the Helmholtz equation in Cartesian coordinates by factoring out a fast varying phase term in the direction. In case where the propagation medium is homogeneous with refractive index $n$, the field component $\Psi$ satisfies the twodimensional scalar wave equation given by

$$
\frac{\partial^{2} \psi}{\partial x^{2}}+\frac{\partial^{2} \psi}{\partial z^{2}}+k^{2} n^{2} \psi=0
$$

where $k$ is the wave number in vacuum. In general, the refractive index varies with direction $\mathrm{x}$ and height $\mathrm{z}$. In the case of small variations of this index, (1) it can be used as a good approach. Considering the electric field component $(E)$ as solution of (1), we introduce it as the reduced function associated with the paraxial direction $x[5]$

$$
E(x, z)=e^{-j k x} \psi(x, z)
$$

The importance of using this reduced function is that it is of slow variation in the direction of the propagation of the energy at angles close to the paraxial direction, which is convenient for property numerical.

The scalar wave equation in terms of electric filed component $E$ is

$$
\frac{\partial^{2} E}{\partial z^{2}}+\frac{\partial^{2} E}{\partial x^{2}}+2 j k \frac{\partial E}{\partial x}+k^{2}\left(n^{2}-1\right) E=0
$$

In the general case, the term $n^{2}$ is a variable depending on the index of refraction of the atmosphere and the surface roughness, but for free-space without boundaries $n^{2}=1$. Equation (1) is factored into two equations for computing the forward and backward propagation waves [6], [7],

$$
\frac{\partial}{\partial x}=-i k(1-Q) E \quad \text { and } \quad \frac{\partial}{\partial x}=-i k(1+Q) E
$$

where $Q=\sqrt{\frac{1}{k^{2}} \frac{\partial^{2}}{\partial z}+n^{2}(x, z)}$.

In this case only one piece is solved giving the propagation in the forward or positive direction.

Using the first order Taylor series and substituting in (4a) we have a standard parabolic equation (SPE) given by [8]

$$
\frac{\partial^{2} E}{\partial z^{2}}(x, z)+2 i k \frac{\partial E}{\partial x}(x, z)+k^{2}\left(n^{2}(x, z)-1\right) E(x, z)=0
$$

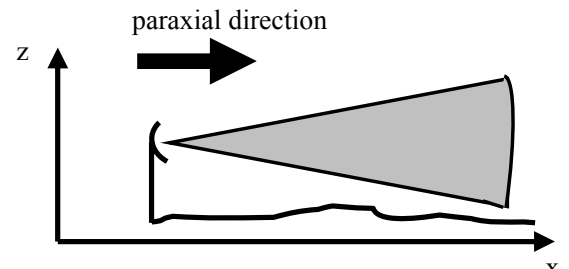

Figure. 1. Paraxial propagation at angles close to the horizontal

In order to solve the Parabolic Equation numerically, (5) can be expressed using finite differences. This is done using the Crank-Nicholson scheme, which permits the modeling of arbitrary boundaries [8]. In this scheme the Parabolic Equation is written using finite differences, which are centered, on a point midway between two successive planes of field points. Using in (5) the equation for the central 
finite-difference approximation of the first-order derivative and second-order derivative, where the midpoint to advance the solution from range $x_{m-1}$ to range $x_{m}$ is $\xi_{m}=\frac{x_{m-1}+x_{m}}{2}, E_{j}^{m}=E\left(x_{m}, z_{j}\right), b=4 i k \frac{\Delta z^{2}}{\Delta x}$ and $a_{j}^{m}=k^{2}\left(n^{2}\left(\xi_{m}, z_{j}\right)-1\right) \Delta z^{2}$, it is obtained

$$
E_{j}^{m}\left(-2+b+a_{j}^{m}\right)+E_{j+1}^{m}+E_{j-1}^{m}=E_{j}^{m-1}\left(2+b-a_{j}^{m}\right)-E_{j+1}^{m-1}-E_{j-1}^{m-1}
$$

for $j=1, \ldots, N-1$. the solution of (6) it is not found explicitly, however it is found implicitly, because it is necessary to invert the matrix to obtain $E$ for the range $x_{m}$. This is the implicit finite difference scheme of Crank - Nicolson type [8].

\section{Path loss models}

To test the efficacy and accuracy of the proposed method, the forward-propagating waves calculated by this algorithm are compared with a measurement campaign and path-loss predictions for some classical models in the literature as described below.

\subsection{Blomquist-Ladell’s Method}

This method considers the free-space loss $\left(L_{0}\right)$, plane-earth loss $\left(L_{p}\right)$, and knife-edge loss $\left(L_{k}\right)$ combined to give the total loss $(L)$ as follows [9]:

$$
L=L_{0}+\left[\left(L_{P}-L_{0}\right)^{2}+L_{D}^{2}\right]^{1 / 2} \approx L_{0}+L_{D}
$$

where

$$
\begin{aligned}
& L_{0}=-147.56-10 \log G_{T}-10 \log G_{R}+20 \log f+20 \log d \\
& L_{D}=-10 \log G_{T}-10 \log G_{R}-20 \log h_{T}-20 \log h_{R}+40 \log d
\end{aligned}
$$

where $f, G_{T}$ and $G_{R}$ was described in (8), $h_{T}$ and $h_{R}$ are the transmitter and mobile receiver height and $d$ is the distance between the transmitter and mobile receiver. The model applies to frequencies within the range $30-$ $900 \mathrm{MHz}$ and distances $5-22 \mathrm{~km}$ range.

\subsection{Multirays's Model}

The modeling of structures with abrupt relief discontinuity can be analyzed by the diffraction and/or reflection in environment components. The multirays's model, based on the study of the diffraction in a knife-edge, it can be used to model that structure type.

\subsubsection{The Two Rays Model}

Figure 2 shows the geometry of the propagation environment studied where two knife-edges are considered. The first is located in point $\mathrm{A}$ and the second in point B associated with the first and second discontinuity of the forest, respectively. Also, it is showed the situation where only two rays are considered as the most important components of the received field [10]. 


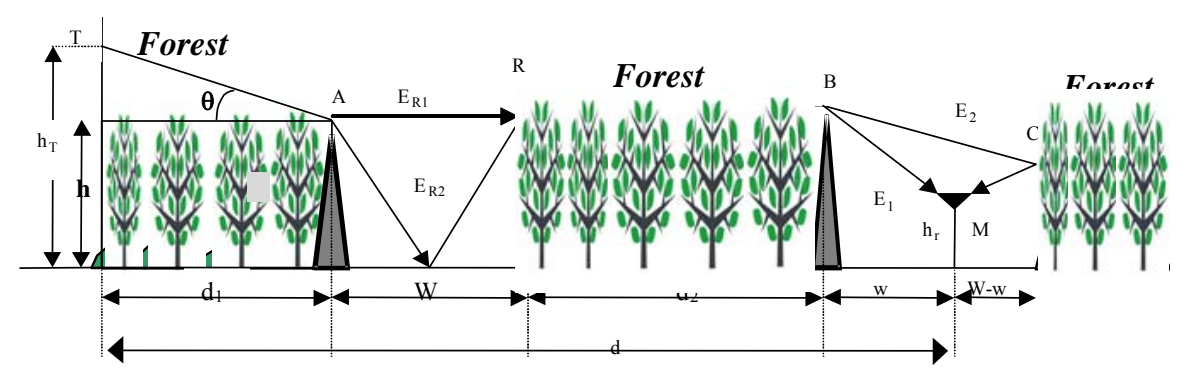

Figure. 2. Geometry of the model (lateral view of the forest)

The mobile receiver field can be calculated as follows [10]:

$$
E_{\text {mobile }}=0.225 \cdot \frac{\sqrt{30 \cdot P_{T} \cdot G_{T}}}{d} \cdot \frac{\left(d_{2}+w\right)}{\left(d_{1}+W\right)} \cdot \sqrt{5+\left(\frac{R_{R O A D}}{v_{R}}\right)^{2}} \cdot \sqrt{\frac{\left(\frac{1}{v_{B M}}\right)^{2}+\left(\frac{R_{F O R E S T}}{v_{B C M}}\right)^{2}}{\left(\frac{1}{v_{B M}^{\prime}}\right)^{2}+\left(\frac{R_{F O R E S T}}{v_{B C M}^{\prime}}\right)^{2}}}
$$

where $R_{F O R E S T}$ and $R_{R O A D}$ are the Fresnel reflection coefficient at forest and road; and $v_{R}, v_{B M}, v_{B C M}, v_{B M}^{\prime}$ and $v^{\prime}{ }_{B C M}$ are the Fresnel parameters.

From (9) we can obtain the path loss

$$
L=20 \log \left(\frac{E_{\text {mobile }}}{E_{0}}\right)
$$

\subsection{Okumura-Hata's Model}

Okumura's predictions of median path loss are usually calculated using Hata's approximations for urban areas, suburban areas, and open areas. The prediction for suburban areas (village or highway scattered with trees and houses, some obstacles near the mobile but not very congested) is calculated as follows [11]

$$
L_{d B}=A+B \log R-C
$$

where

$$
\begin{aligned}
& A=69.55+26.16 \log f_{c}-13.82 \log h_{T} \\
& B=44.9-6.55 \log h_{T} \\
& C=2\left(\log \left(f_{c} / 28\right)\right)^{2}+5.4
\end{aligned}
$$

$R$ is the great circle distance between base station and mobile and $f_{c}$ is the carrier frequency. The model is valid only for $150 \mathrm{MHz} \leq f_{c} \leq 1500 \mathrm{MHz}, 30 \mathrm{~m} \leq h_{T} \leq 200 \mathrm{~m}$, and $R>1 \mathrm{~km}$. 


\section{Results}

The measurement environment is showed in Figure 3. The measurements were taken in a covered by radio signal transmitted by fixed station in the surroundings of Belém, state of Pará in a town called Benfica. The transmitted signal was at $900 \mathrm{MHz}$, using a collinear antenna in a twelve-height tower. The transmitter was installed on a building of the ANATEL (Agencia Nacional de Telecomunicações). The mobile receiver has traveled along a $5.6 \mathrm{~km}$ road inside a forest at a speed of approximately $30 \mathrm{~km} / \mathrm{h}$. The measurement results were recorded for off line processing. The map of measurement environment (Figure 3a) indicates the transmitter position, and the path of mobile receiver unit in road. Figure $3 \mathrm{~b}$ shows a view of the road crossing forest.

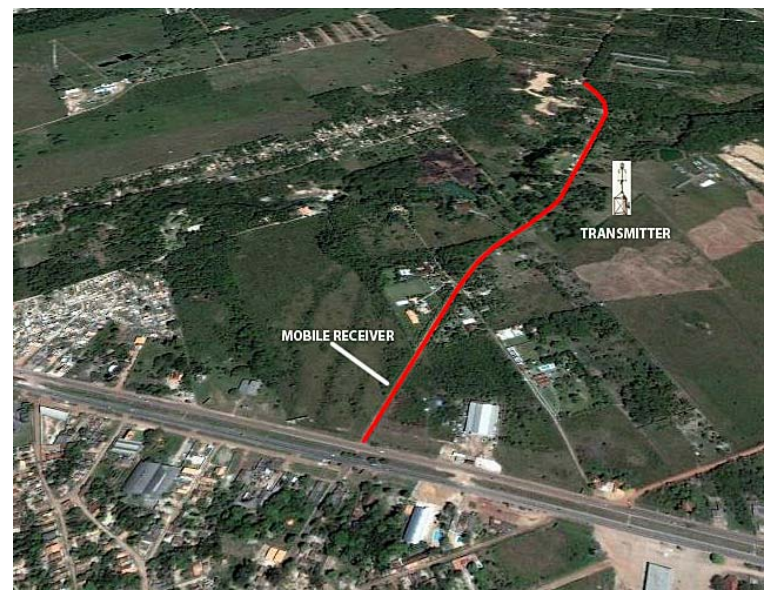

(a) Map of Benfica road inside forest

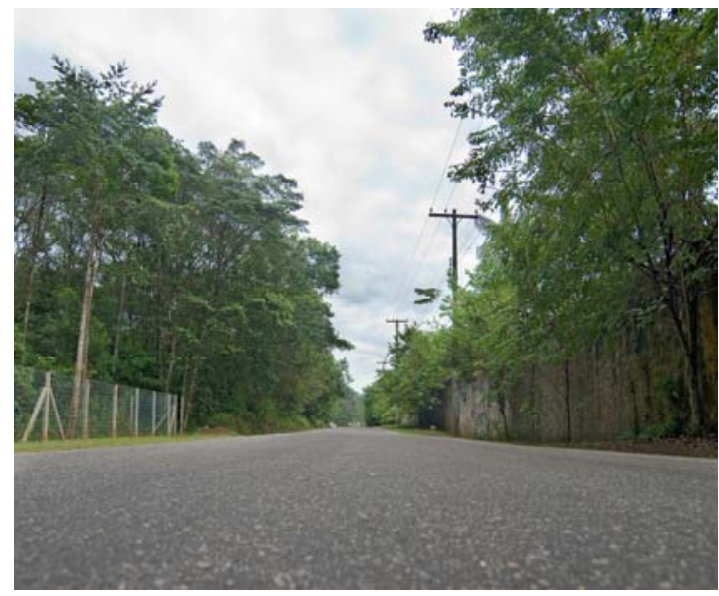

(b) Benfica road

Fig. 3. Measurement environment

A plane wave vertically polarized in $900 \mathrm{MHz}$ was transmitted for simulation using the parabolic equation method. The paraxial direction (Figure 1) was chosen, with approach for small angles, up to $15^{\circ}$. Also was considered only the dimensions of length and width of vegetation and road in studied environments. The refractive index was considered complex as given by [12]. A FORTRAN program was developed to simulate (6). In order to compare the Parabolic Equation method with measured data and the path los models the electric field given by (6) was introduced in (13) to obtain the path loss.

$$
L(d B)=36,57+20 \log _{10} f+20 \log _{10}\left|E_{0}\right|-20 \log _{10}|E|-G_{T}-G_{R}
$$

where $E_{0}$ is the field in the distance of reference $\left(\mathrm{d}_{0}\right), E$ is the received field, $f$ is the frequency in $\mathrm{GHz}$, and $G_{T}$ and $G_{R}$ are the transmitter and receiving antennas gain in $\mathrm{dB}$, respectively [13].

The parameters showed in the Table 1 were used to simulate the models considered.

Table 1. Parameters used in the models

\begin{tabular}{|c|c|c|}
\hline Parameters & Symbols & Values \\
\hline Frequency & $f$ & $900 \mathrm{MHz}$ \\
\hline Average height forest & $h$ & $12 \mathrm{~m}$ \\
\hline Transmitter height & $h_{T}$ & $12 \mathrm{~m}$ \\
\hline Mobile receiver height & $h_{R}$ & $3 \mathrm{~m}$ \\
\hline Transmitter antenna gain & $G_{T}$ & $2.14 \mathrm{~dB}$ \\
\hline Receiver antenna gain & $G_{R}$ & $2.14 \mathrm{~dB}$ \\
\hline Transmitted power & $P_{T}$ & $30 \mathrm{dBm}$ \\
\hline Road - paved, width & $W$ & $12 \mathrm{~m}$ \\
\hline Vehicle position & $w$ & $7.75 \mathrm{~m}$ \\
\hline Forest relative permittivity [10] & $\varepsilon_{F}$ & 1.1 \\
\hline
\end{tabular}




\begin{tabular}{|c|c|c|}
\hline Parameters & Symbols & Values \\
\hline Forest conductivity [10] & $\sigma_{F}$ & $0.1 \mathrm{mS} / \mathrm{m}$ \\
\hline Road relative permittivity [10] & $\varepsilon_{R}$ & 2.7 \\
\hline Road conductivity [10] & $\sigma_{R}$ & $40 \mathrm{mS} / \mathrm{m}$ \\
\hline Width of the first forest & $d_{1}$ & $1500 \mathrm{~m}$ \\
\hline Width of the second forest & $d_{2}$ & $2500 \mathrm{~m}$ \\
\hline Transmitter distance range & $d$ & 500 to $5600 \mathrm{~m}$ \\
\hline
\end{tabular}

The Figure 4 shows the path loss, in decibels, as a function of distance $d$, in meters, in the representative case. This result is compared with the model proposed by Cavalcante et all, called Multirays, with the Blomquist-Ladell's Method, with the Okumura-Hata's Model, and with the measured. In the Figure 4 we can observe a best fit to the experimental data by proposed method in this work.

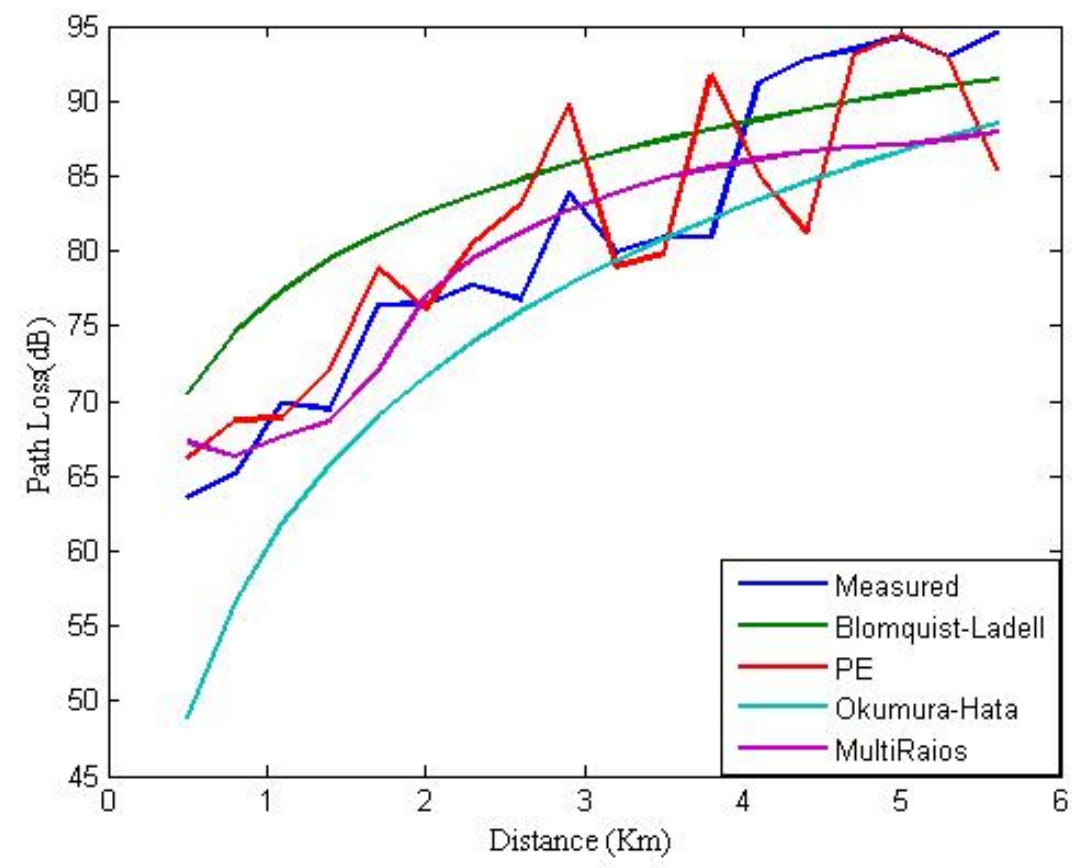

Figure 4. Path loss with distance of the theoretical and experimental results

The Table 2 shows the average error, standard deviation and rms error for several models, compared with the measured, for the test environment studied.

Table 2. Average error, standard deviation and rms error

\begin{tabular}{|c|c|c|c|}
\hline Model & $\begin{array}{c}\text { Average error } \\
\text { (dB) }\end{array}$ & $\begin{array}{c}\text { Standard } \\
\text { deviation (dB) }\end{array}$ & $\begin{array}{c}\text { Rms error } \\
\text { (dB) }\end{array}$ \\
\hline PE method & 3.40 & 2.75 & 4.37 \\
\hline Blomquist-Ladell [9] & 5.51 & 3.08 & 6.31 \\
\hline Multirays [10] & 3.86 & 2.17 & 4.43 \\
\hline Okumura-Hata [11] & 5.71 & 4.15 & 7.06 \\
\hline
\end{tabular}




\section{Conclusion}

Several theoretical and experimental models exist for to compute the path loss propagation in wireless communications, and each characterizes the environment with different view. In mixed-path, the shadowing, the scattering and the absorption caused for the vegetation can cause a significant path loss, which increases with the frequency.

This work shows that parabolic equation method can be applied to the electromagnetic waves propagation in forest environment. The principle of the domain decomposition technique for parabolic equation was introduced. Good agreements between the measured and simulated results through of parabolic equation method are also achieved. The limitations of the standard parabolic equation approximation are due to its bad behavior at large propagation angles.

An advantage of the parabolic equation method is the reduction of the computational time in relation to the similar techniques found in the literature. This is due to the fact that the problem is reduced to a tridiagonal system and the method to solve such system is the implicit finite differences scheme of the CrankNicolson type. This is a method stable unconditionally, what allows the change of the parameters of the middle without gets lost the stability. The adopted methodology allows the incorporation of new elements in geometric ways different with their respective properties electric.

Actually, we are made simulation increasing the angle of propagation with the paraxial direction, for $1.8 \mathrm{GHz}$ that will be applied for this and other environments.

\section{References}

[1] Whitman, G. M., F. K. Schwering, and M. Y.-C. Wu, "Collimated Beam Wave Pulse Propagation and Scattering in Vegetation Using Scalar Transport Theory", IEEE Transactions on Antennas and Propagation, Vol. 55, No. 6, pp. 1599-1612, June 2007.

[2] Wang, F., and K. Sarabandi, "A Physics-Based Statistical Model for Wave Propagation Through Foliage", IEEE Transactions on Antennas and Propagation, Vol. 55, No.3, pp. 958-968, March 2007.

[3] Valtr, P., and P. Pechac, "Domain Decomposition Algorithm for Complex Boundary Modeling using the Fourier Split-Step Parabolic Equation”, IEEE Antennas and Wireless Propagation Letters, Vol. 6, pp. $152-155,2007$.

[4] Arshad, K., F. Katsriku, and A. Lasebae, "Radiowave VHF Propagation modelling in forest using finite elements", IEEE, pp.2146-2149, 2006.

[5] Brekhovskikh, L. M., "Waves in Layered Media”, 2nd ed., Academic Press, New York, 1980.

[6] Levy, M. F., and A. A. Zaporozhets, "Target Scattering Calculations with the Parabolic Equation Method", J. Acoust. Soc. Am. Vol. 103, pp. 735-741, 1998.

[7] Mallahzadeh, A. R., M. Soleimamni, and J. Rashed-Mohassel, "RCS computation of Airplane using Parabolic Equation", PIER 57, pp. 151-163, 2006.

[8] Levy, M., "Parabolic Equation Methods for Electromagnetic Wave Propagation", The Institution of Electrical Engineers, London, 2000.

[9] Yacoub, M. D., "Foundations of Mobile Radio Engineering", CRC Press, United States of America, 1993.

[10] Cavalcante, G. P. S., M. A. R. Sanches, and R. A. N. Oliveira, "Mobile Radio Propagation along Mixed Paths in Forest Environment”, Proceedings of IMOC'99, pp. 320-324, SBMO/IEEE, 1999.

[11] Saunders, S. R., “Antennas and Propagation for Wireless Communication Systems", John Wiley \& Sons, England, December 1999.

[12] Wangsness, R. K., "Electromagnetic Fields", John Wiley \& Sons, United States of America, pp. 34 appendices, 1979.

[13] Li, L. W., T. S. Yeo, P. S. Kooi, M. S. Leong, and J. H. Koh, "Analysis of Electromagnetic Wave Propagation in Forest Environment along Multiple Paths", Progress In Electromagnetics Research, PIER 23, pp. 137-164, 1999. 